\title{
Pulmonary Synovial Sarcoma: An Unpleasant Surprise
}

\author{
Gabriela Pereira, Ana Pires, Sandra Barbosa, Jorge Cotter \\ Hospital Senhora da Oliveira, Guimarães, Portugal
}

Received: 03/01/2021

Accepted: 20/01/2021

Published: $16 / 02 / 2021$

How to cite this article: Pereira G, Pires A, Barbosa S, Cotter J. Pulmonary synovial sarcoma: an unpleasant surprise. EJCRIM 2021;8: doi:10.12890/2021_002149.

Conflicts of Interests: The Authors declare that there are no competing interests.

This article is licensed under a Commons Attribution Non-Commercial 4.0 License

\section{ABSTRACT}

The authors describe the case of a patient with primary monophasic pulmonary synovial sarcoma presenting as a right pleural effusion, and discuss the underlying diagnostic challenges.

\section{LEARNING POINTS}

- There has been tremendous progress in the diagnosis and management of malignant effusion, with thoracoscopic biopsy of the pleura providing the highest diagnostic yield.

- Even though advanced techniques such as image-guided biopsy and pleuroscopy have relatively high sensitivity and specificity, in rare cases, the diagnosis can still be missed.

- In clinical practice, all such techniques should be considered in order to achieve an early diagnosis and a better outcome.

\section{KEYWORDS}

Sarcoma, pleural effusion, neoplasm, monophasic

\section{INTRODUCTION}

Primary intrathoracic synovial sarcoma is a very uncommon, highly aggressive malignant neoplasm, representing $<0.5 \%$ of lung tumours, and equally affecting both genders, mostly young adults, with no preference for any specific part of the lung ${ }^{[1]}$.

Morphological, histochemical and cytogenetic features are similar to the other soft tissue synovial sarcomas, so the approach and treatment strategies are the same. Histologically, the most common subtypes are the biphasic, monophasic spindle cell or fibrous, monophasic epithelial, and poorly differentiated subtypes. The monophasic type is difficult to diagnose because it has a uniform spindle cell pattern, indistinguishable from other malignant spindle cell neoplasms; therefore, immunohistochemistry is essential when making a differential diagnosis.

The gold standard for definitive diagnosis is detection of a chromosomal translocation producing the SS18-SSX fusion gene, present in more than $90 \%$ of cases of synovial sarcomas, since often only focal changes within an epithelial-like nodule are noticeable, sometimes even with a benign appearance, which can easily be misdiagnosed ${ }^{[1,2]}$.

There are no specific clinical or radiological features pathognomonic of pulmonary synovial sarcoma, which may create diagnostic challenges $^{[2]}$.

\section{CASE DESCRIPTION}

A 52-year-old man had been admitted for epigastric discomfort associated with anorexia and a 7\% weight loss in the previous 2 months. Hence, he had undergone upper gastrointestinal endoscopy, which revealed acute gastritis with Helicobacter pylori infection, for which he 
had received eradication treatment for 14 days. However, symptoms did not improve, and 1 month later, he developed chest pain with pleuritic characteristics in the right hemithorax. The pain was of medium intensity, intermittent, and had worsened over the last 48 hours. He denied other pain complaints, chest trauma, fever, nausea, vomiting, dyspnoea, cough, sputum, nocturnal hyperhidrosis, and gastrointestinal tract or genitourinary system symptoms. His medical history included pulmonary tuberculosis at age 21 (with good response to 6-month treatment) and psoriasis. The patient was a non-smoker, denied alcohol consumption, and had no known toxic exposures or any family history of malignancies.

Imaging confirmed a moderate pleural effusion with slight parenchymal consolidation associated with a probable infectious process at the right lung base (Fig. 1). The pleural fluid analysis was compatible with exudate and showed a predominance of polymorphonuclear cells (Table 1). We started antibiotic treatment with ceftriaxone and azithromycin and an exhaustive study of possible aetiologies.

Blood cultures, bacterial and mycological examination of sputum and pleural fluid, and viral serologies were negative. However, after 6 days, the patient's clinical condition worsened, so it was decided to escalate antibiotics (meropenem) and perform a new thoracentesis that showed an exudate now with a predominance of mononuclear cells (Table 2).

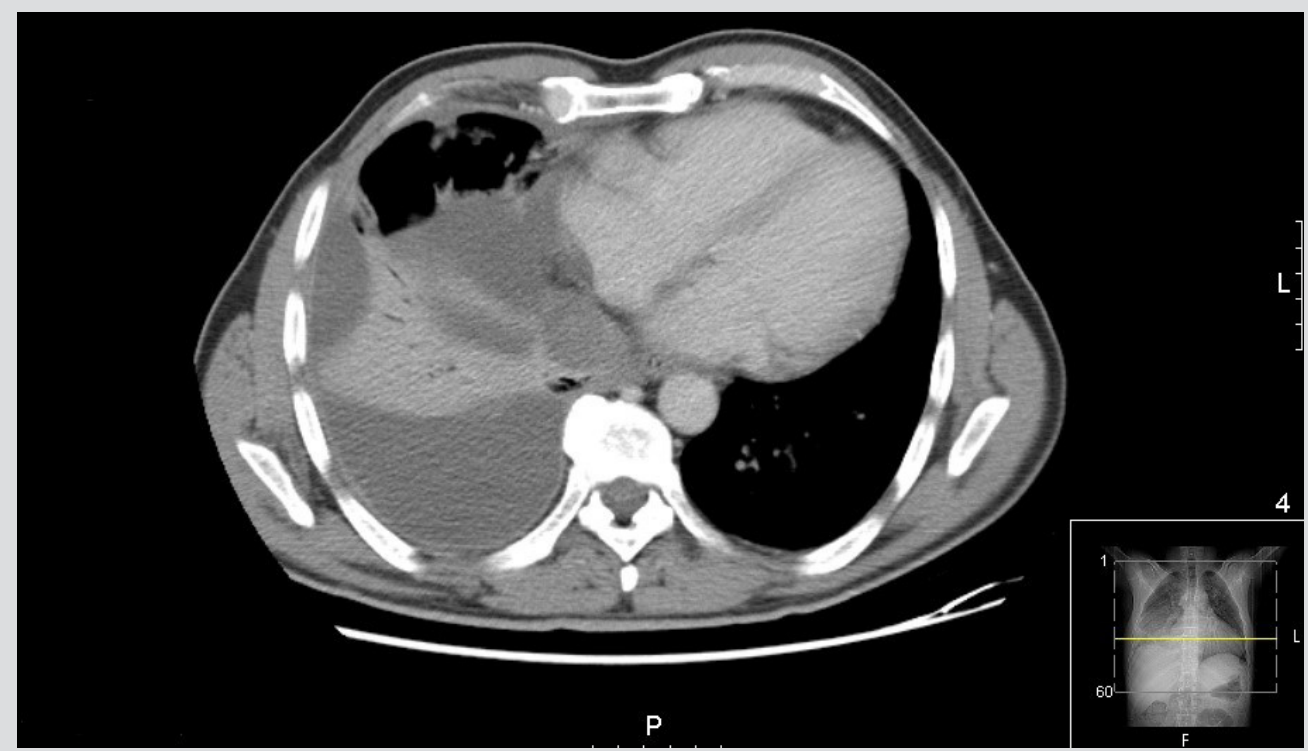

Figure 1. Chest CT scan (axial): medium volume pleural effusion with parenchymal densification on the right

\begin{tabular}{|l|l|}
\hline \multicolumn{2}{|c|}{ Pleural Fluid Analysis } \\
\hline$p H$ & 7.5 \\
\hline Neutrophils (\%) & 74.4 \\
\hline Lymphocytes (\%) & 25.6 \\
\hline WBC (//l) & $17,122(<1,000)$ \\
\hline ADA (IU/I) & $27.6(<60)$ \\
\hline Pleural LDH/serum LDH & 2.2 \\
\hline Pleural/serum total protein ratio & 0.72 \\
\hline Glucose (mg/dl) & 80 \\
\hline
\end{tabular}

Table 1. Pleural fusion analysis following the first diagnostic thoracentesis $A D A$, adenosine deaminase; $L D H$, lactate dehydrogenase; WBC, white blood cell count

\begin{tabular}{|l|l|}
\hline \multicolumn{2}{|c|}{ Pleural Fluid Analysis } \\
\hline$p H$ & 7.5 \\
\hline Neutrophils (\%) & 44.6 \\
\hline Lymphocytes (\%) & 55.4 \\
\hline WBC (/ul) & $7,257(<1,000)$ \\
\hline ADA (IU/I) & $25.4(<60)$ \\
\hline Pleural LDH/serum LDH & 2.2 \\
\hline Pleural/serum total protein ratio & 0.76 \\
\hline Glucose (mg/dl) & 115 \\
\hline
\end{tabular}

Table 2. Pleural fusion analysis following the second diagnostic thoracentesis $A D A$, adenosine deaminase; $L D H$, lactate dehydrogenase; WBC, white blood cell count 
The abdominopelvic, axillary, cervical and inguinal ultrasound scans showed no alterations. The interferon-gamma release assay (IGRA) was negative. An immunoglobulin assay was normal. Flow cytometry immunophenotyping of pleural fluid cytology showed a predominance of small lymphocytes without immunophenotypic changes suggestive of lymphoproliferative disease. An echo-guided biopsy was performed, and histology showed unspecific chronic pleuritis. Polymerase chain reaction (PCR) for detection of Mycobacterium tuberculosis in pleural fluid and sputum was negative.

Since the patient maintained fever and chest pain despite the administration of antibiotics, he underwent pleuroscopy on the 25th day, which could not be completed because of extensive pleural adhesions. Therefore, it was decided at a multidisciplinary meeting to place a thoracic drain to drain the effusion that had become even more loculated. A CT scan suggested possible empyema (Fig. 2). Bronchofibroscopy was then carried out but showed no relevant changes. A new echo-guided biopsy was performed and showed only non-specific reactive pleuritis. Over the following 15 days, the patient maintained sustained apyrexia and clinical stability. However, pleural drainage was scarce (110 ml in total) and serohematic, without significant imaging improvement, so intrapleural alteplase was administered for adhesion lysis (Fig. 3). Meanwhile, serial tests of bacterial sputum cultures were conducted and Mycobacterium avium was isolated.

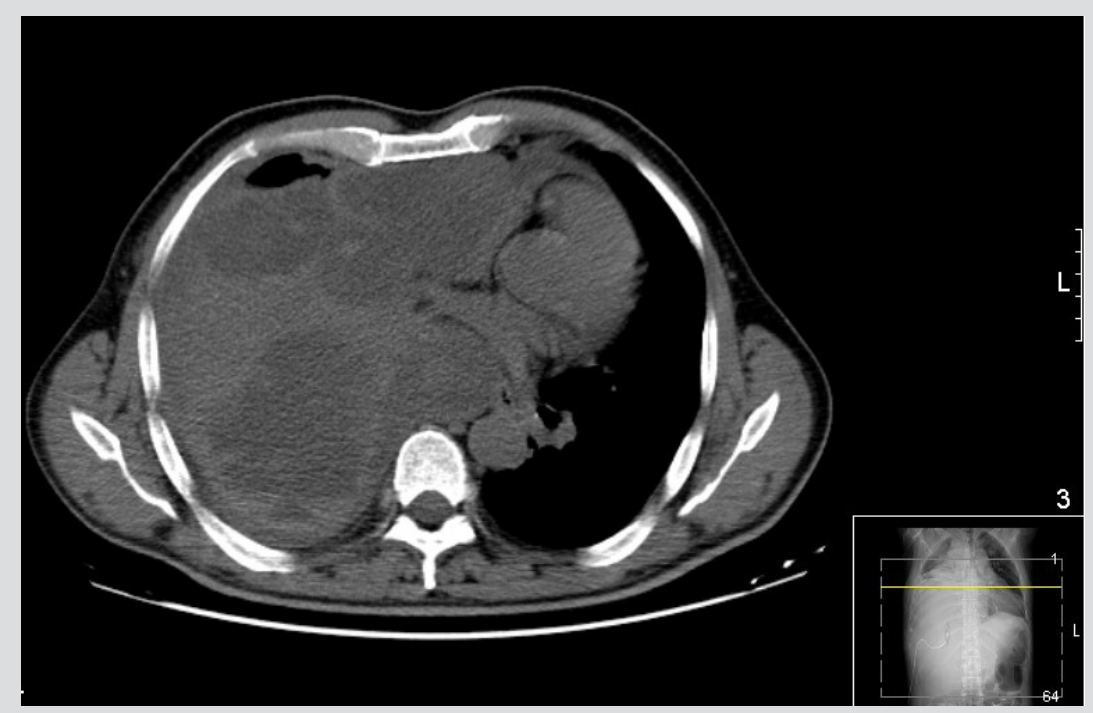

Figure 2. Chest CT scan (axial): large pleural effusion on the right, with signs of some organization, with loculation and enhancement of the pleural leaflets. The hyperdense areas within the pleural effusion indicate dense/haemorrhagic content

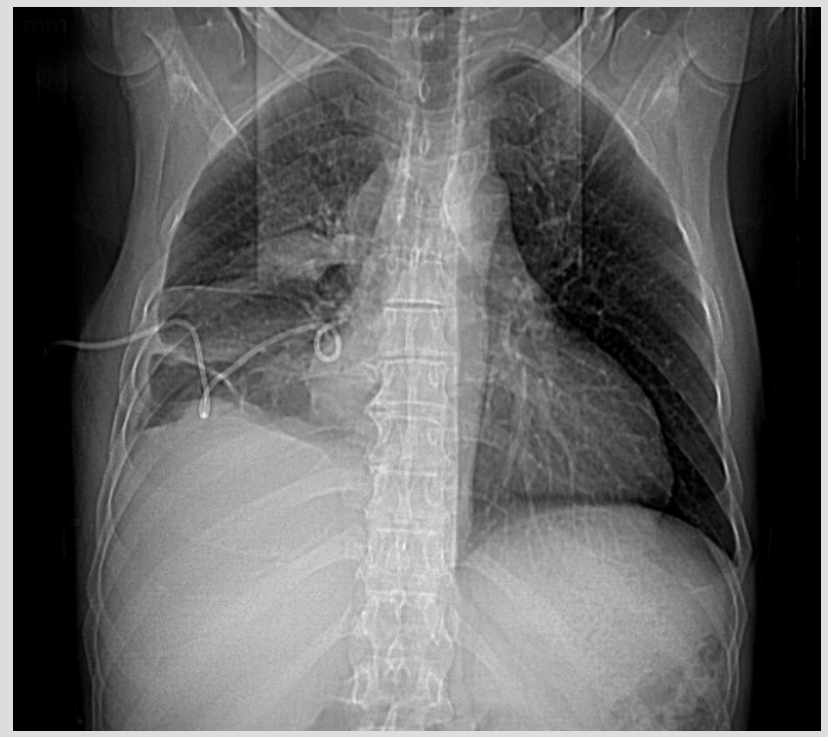

Figure 3. Chest $x$-ray after administration of intrapleural alteplase

On the 50th day, the patient was again febrile and showed no clinical or radiological improvement, so treatment for M. avium was started. Initially, there was a slight clinical improvement, but then the patient's respiratory symptoms began to worsen with trepopnoea, dyspnoea on minor effort and prostration. It was decided to perform a pulmonary thoracotomy for decortication and drainage of pleural effusion, which was completed without complications, with clinical and imaging improvement (Fig. 4). 
Nevertheless, the patient again worsened (Fig. 5). We received the results of histological examination of the surgical specimen which showed a malignant fusocellular neoplasm, compatible with monophasic synovial sarcoma. Despite supportive treatment, the patient became haemodynamically unstable and eventually died before it was possible to perform cytogenetic analysis to define treatment strategy.
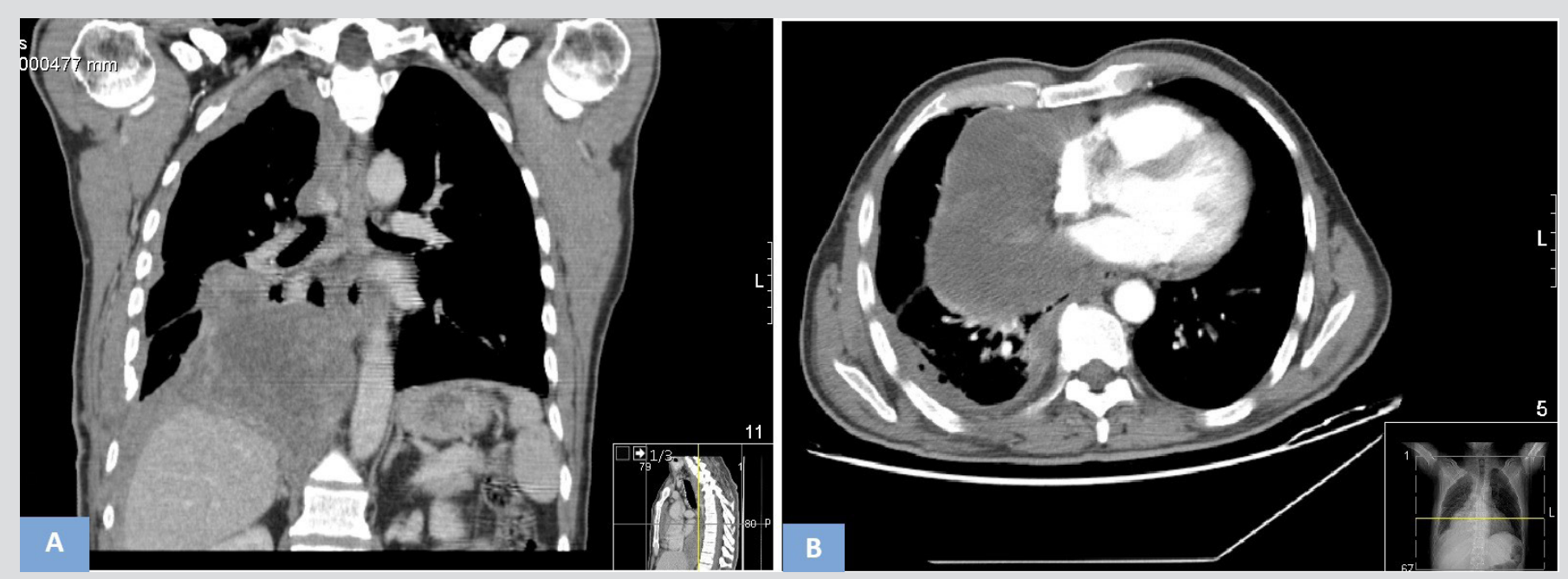

Figure 4. Chest CT scan (A - coronal, B - axial): heterogeneous right pleural effusion, with associated air bubbles (iatrogenic - after thoracotomy), with lobulated aspect
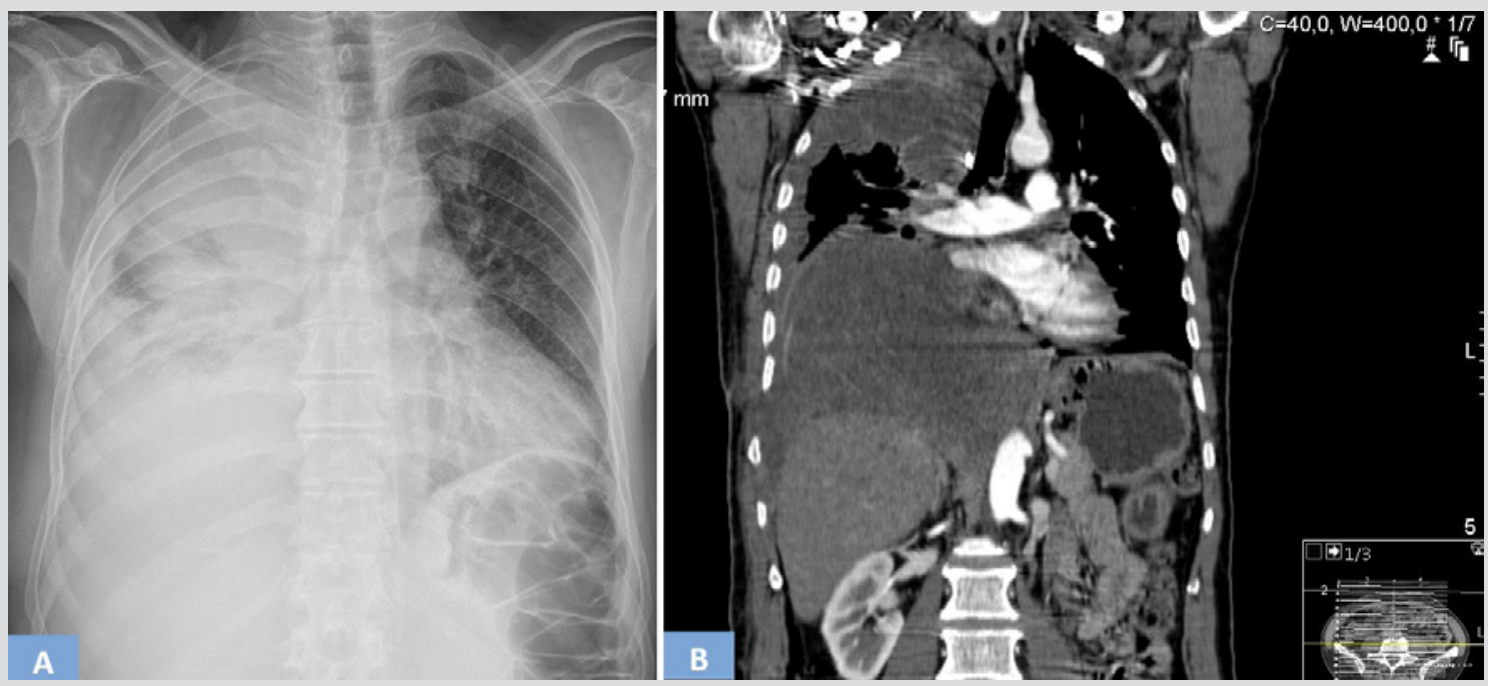

Figure 5. (A) Chest $x$-ray and (B) chest CT scan: deviation of the mediastinum to the left, in relation to a right pleuroparenchymal lesion, compatible with a neoplastic lesion that causes almost complete collapse of the right lung

\section{DISCUSSION}

Pulmonary synovial sarcoma is an extremely rare intrathoracic tumour, with a median age of presentation slightly greater than that of soft tissue synovia sarcomas. Bone invasion or mediastinal adenopathy is unusual ${ }^{[2]}$.

There are two main histological types, monophasic (most common) and biphasic, which both show features of poor differentiation. Immunohistochemically, synovial sarcomas are nearly uniformly positive for cytokeratin, EMA, bcl-2 and vimentin, and negative for S-100, desmin, smooth muscle actin, and vascular tumour markers. Chromosomal translocation producing the SS18-SSX fusion gene is present in more than $90 \%$ of cases $^{[2]}$.

As for clinical presentation, chest pain is the most common symptom, although pleural effusion, dyspnoea, dysphagia and/or pneumothorax may also occur. Also, many patients can be asymptomatic, so diagnosis is an incidental finding ${ }^{[2]}$. 
The case reported here was a clinical challenge, as multiple confounding factors limited the fast and early diagnosis of the malignant neoplasm. What initially appeared to be just a parapneumonic effusion become a worrying situation when the patient's condition deteriorated. Pleural fluid cytology, which is reported to have a mean sensitivity of about $72 \%$, was repeatedly negative ${ }^{[3]}$.

As recommended in cases of non-diagnostic thoracentesis and in the presence of lymphocytic exudate, a definitive diagnosis is established by histopathological analysis of samples obtained by non-image-guided or image-guided closed pleural biopsy (CPB), with these methods having a diagnostic yield of $38-47 \%$ and $75-88 \%$ respectively, or by video-assisted thoracoscopy, which has a diagnostic yield of $95-97 \%$. In relation to tuberculous lesions, CPB has a sensitivity of $66.7 \%$ and a specificity of $100 \%{ }^{[3,4]}$.

Since videothoracoscopy is not available in our hospital, the patient initially underwent image-guided CPB, which only showed chronic pleuritis. The patient lived in an area endemic for tuberculosis, but all culture tests and PCR for M. tuberculosis in pleural fluid and sputum were negative, IGRA was negative and CPB, which has a relatively high sensitivity and specificity for tuberculous lesions, did not show any alterations compatible with tuberculosis granulomas. Therefore, it was decided to adopt a wait-and-see approach and not empirical treat possible pleural tuberculosis.

After clinical worsening, the patient was transferred to another hospital for pleuroscopy, but the procedure failed because of the multiple pleural adhesions in the pleural cavity, which suggested the need for thoracotomy. However, as the patient was stable, it was decided at a multidisciplinary meeting to place a thoracic drain and perform a new image-guided CPB, which was inconclusive and may have delayed the diagnosis.

Despite the therapeutic effort and various attempts at diagnosis using invasive methods, pleural biopsy and thoracoscopy, which strongly suggested malignancy, the final diagnosis of a rapidly progressive malignant neoplasm was only confirmed through examination of a surgical specimen obtained during thoracotomy.

The tumour behaviour of synovial sarcomas remains unpredictable and they are considered high-grade tumours with a poor prognosis (overall 5 -year survival rate of 50\%). There is no standardized therapy, which may include surgical treatment, chemotherapy and radiotherapy. There are no controlled studies of adjuvant chemotherapy due to the rarity of these tumours. Synovial sarcomas are chemosensitive to ifosfamide and doxorubicin, with an overall response rate of approximately $24 \%{ }^{[5]}$.

\section{REFERENCES}

1. Panigrahi MK, Pradhan G, Sahoo N, Mishra P, Patra S, Mohapatra PR. Primary pulmonary synovial sarcoma: a reappraisal. J Cancer Res Ther 2018;14(3):481-489.

2. Etienne-Mastroianni B, Falchero L, Chalabreysse L, Loire R, Ranchère D, Souquet PJ, et al. Primary sarcomas of the lung: a clinicopathologic study of 12 cases. Lung Cancer 2002;38(3):283-289.

3. Rivera MP, Mehta AC, Wahidi MM. Establishing the diagnosis of lung cancer: diagnosis and management of lung cancer, 3rd ed: American College of Chest Physicians evidencebased clinical practice guidelines. Chest 2013;143(5 Suppl):e142S-e165S.

4. Zhang T, Wan B, Wang L, Li C, Xu Y, Wang X, et al. The diagnostic yield of closed needle pleural biopsy in exudative pleural effusion: a retrospective 10-year study. Ann Transl Med 2020;8(7):491.

5. Vlenterie M, Jones RL, van der Graaf WT. Synovial sarcoma diagnosis and management in the era of targeted therapies. Curr Opin Oncol $2015 ; 27(4): 316-322$. 